\section{(6) OPEN ACCESS}

\title{
Two-year outcomes of intravitreal bevacizumab therapy for macular oedema secondary to branch retinal vein occlusion
}

\author{
Taiichi Hikichi, Makoto Higuchi, Takuro Matsushita, Shoko Kosaka, Reiko Matsushita, \\ Kimitaka Takami, Hideo Ohtsuka, Hirokuni Kitamei, Shoko Shioya
}

Department of Ophthalmology, Ohtsuka Eye Hospital, Sapporo, Japan

\section{Correspondence to} Dr Taiichi Hikichi, Department of Ophthalmology, Ohtsuka Eye Hospital, Kita-16 Nishi-4, Kita-ku, Sapporo 001-0016, Japan; taiichi-hikichi@ hokkaido.med.or.jp

Received 15 January 2013 Revised 6 August 2013 Accepted 15 September 2013 Published Online First 8 November 2013

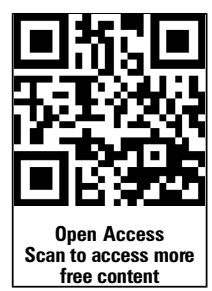

To cite: Hikichi T, Higuchi M, Matsushita T, et al. Br J Ophthalmol 2014;98:195-199.

\section{ABSTRACT}

Aim To determine the 2-year outcomes of intravitreal bevacizumab (IVB) injections in eyes with macular oedema (ME) following branch retinal vein occlusion (BRVO).

Methods of 105 consecutive eyes (105 treatmentnaïve patients) with ME following BRV0, 89 eyes were followed for 2 years after the first injection. During the 2-year follow-up period, patients were examined at least every 3 months and received an IVB injection $(1.25 \mathrm{mg} /$ $0.05 \mathrm{~mL}$ ) if they met prespecified retreatment criteria. Rescue grid laser was permitted based on the findings of the Branch Vein Occlusion Study.

Results The baseline logarithm of the minimum angle of resolution visual acuity (VA) was $0.64 \pm 0.24$ (mean $\pm S D)$, which significantly $(p=0.001)$ improved 1 month after the first injection to $0.39 \pm 0.22$. One year after the first injection, VA improved significantly $(p=0.001)$ to $0.33 \pm 0.21$ and remained $0.34 \pm 0.21$ until 2 years after the first injection $(p=0.001)$. The changes in foveal thickness were correlated with those of VA during the 2year follow-up period with a mean of $3.8 \pm 1.5$ injections (including the first injection).

Conclusions This relatively large case series study showed favourable 2-year outcomes using bevacizumab to treat ME following BRVO. Bevacizumab provides substantial long-term benefits in the treatment of ME following BRVO.

\section{INTRODUCTION}

Macular oedema (ME) is the main cause of decreased visual acuity (VA) in branch retinal vein occlusion (BRVO). In 1984, a randomised, controlled study ${ }^{1}$ reported that grid photocoagulation of ME following BRVO resulted in better visual improvement than during the natural course of the disease. In 2009, the Standard Care versus Corticosteroid for Retinal Vein Occlusion (SCORE) study $^{2}$ found that $29 \%$ of eyes treated with laser photocoagulation gained 15 or more letters of VA measured using the Early Treatment Diabetic Retinopathy Study (ETDRS) ${ }^{3}$ chart at the 1-year primary end point.

The rationale for use of an intravitreally injected antivascular endothelial growth factor (VEGF) drug to treat BRVO is that vascular occlusion induces upregulation of VEGF, resulting in increased vascular permeability and subsequent $\mathrm{ME}^{4-6}$ Recent clinical studies have reported the beneficial effects of anti-VEGF therapy for ME following BRVO. ${ }^{7-18}$ Prospective studies of ranibizumab (Lucentis, Genentech, Inc., South San Francisco, California,
USA), a humanised, affinity-matured VEGF antibody fragment that neutralises all isoforms of VEGF-A and their biologically active degradation products in treatment-naïve eyes with ME following BRVO, found that ranibizumab was effective at 2 years after treatment of ME caused by BRVO. ${ }^{12}$ Bevacizumab (Avastin, Genentech, Inc.), a humanised monoclonal antibody directed against VEGF, is efficacious for treating ME following BRVO. ${ }^{14-18}$ The current study reports the 2-year outcomes in a large number of eyes with ME following BRVO treated with intravitreal bevacizumab (IVB) injections.

\section{PATIENTS AND METHODS}

This study was an open-label, single-arm, singlecentre trial that was conducted in accordance with the Declaration of Helsinki; the institutional review board of Ohtsuka Eye Hospital approved the study protocol before study initiation. The off-label use of bevacizumab was explained to all patients before study enrolment, and all patients provided informed consent. Patients with a decimal VA between 0.8 (20/25 Snellen VA) and 0.05 (20/400 Snellen VA) as a result of treatment-naïve ME secondary to BRVO were eligible if the foveal thickness was $250 \mu \mathrm{m}$ or more and none of the following were present: possible permanent visual loss in the study eye (atrophy or prominent pigmentary macular changes); vitreomacular traction or an epiretinal membrane; a history of vitreous surgery and intravitreal injection of a VEGF antagonist or steroids; or ME in the study eye due to causes other than BRVO, such as diabetic retinopathy.

Eligible patients were evaluated at least every 3 months or more frequently. ${ }^{9}$ At each study visit, patients could receive an IVB injection $(1.25 \mathrm{mg} /$ $0.05 \mathrm{~mL}$ ) if the foveal thickness was $250 \mu \mathrm{m}$ or more or if there was persistent or recurrent $\mathrm{ME}$ that affected the VA based on the investigator's evaluation. ${ }^{9}$ Thus, even if the foveal thickness was less than $250 \mu \mathrm{m}$, an intravitreal injection was administered in eyes in which ME around the fovea persisted or recurred. Patients were examined 1 month after each IVB injection, and if additional treatment was not required, the next visit was planned for 2 months later. Bevacizumab injections were administered under sterile conditions in the operating room.

At baseline and every visit during the follow-up period, all patients underwent a complete ophthalmologic examination, including measurement of 
the best-corrected VA (BCVA) using a Landolt ring VA chart, intraocular pressure (IOP) measurement and macular evaluation with optical coherence tomography (OCT) (OCT 3000, Zeiss Humphrey Instruments, Dublin, California, USA). Six radial line scans through the centre of the foveal lesion were used to determine if fluid was present in the macula. The foveal thickness was defined as the average foveal thickness measured on the vertical and horizontal scans. Fluorescein angiography (FA) was performed in all patients from 3 to 6 months after study entry when most retinal haemorrhages had cleared sufficiently to allow appropriate evaluation of the retinal vascular findings.

Rescue grid laser treatment was allowed based on the findings of a previous study. ${ }^{1}$ Starting at month 3 , patients were eligible for laser treatment if haemorrhages had cleared sufficiently to allow safe laser application and the following criteria were met: a decimal VA of 0.5 or less (20/40 Snellen VA), foveal thickness $250 \mu \mathrm{m}$ or more and fluorescein leakage at the macula on FA.

Statistical analysis was performed using the SPSS software package (SPSS Inc., Chicago, Illinois, USA). The BCVA measured using a Landolt ring chart was converted to the logarithm of the minimum angle of resolution (logMAR) for statistical analyses. The VA and foveal thickness were compared using the paired Student t test. $\mathrm{p}<0.05$ was considered significant.

\section{RESULTS}

A total of 105 consecutive eyes of 105 treatment-naïve Japanese patients with ME that developed after BRVO, who received the first bevacizumab injection between 1 April 2008, and 31 March 2009, were included. Of the 105 eyes, 16 (15\%) were lost to follow-up during the 2 years after the first injection (10 after more than 3 months without recurrent $\mathrm{ME}$ during the second year, 4 after confirmation of $\mathrm{ME}$ resolution after the injection and 2 after reinjection for recurrent ME). Thus, 89 eyes of 89 patients ( 48 men and 41 women) who could be followed for 2 years after the first injection were analysed. The mean $( \pm S D)$ age of the 64 patients was $65 \pm 11$ years (range 46-86).

FA showed no disruption of the perifoveal capillaries in 22 $(25 \%)$ eyes, disruption of $180^{\circ}$ or less in $40(45 \%)$ eyes and disruption of more than $180^{\circ}$ in $27(30 \%)$ eyes. An area of nonperfusion exceeding 10 disc diameters was found in 21 (24\%) eyes.
The mean numbers of injections were 2.6 \pm 0.9 during the first year (included the first injection) and $1.3 \pm 1.0$ during the second year. The mean total number of injections (including the first injection) over 2 years was $3.8 \pm 1.5$. During the first year, $12(13 \%)$ eyes required no additional injection, 27 (30\%) eyes required one injection, 39 (44\%) eyes required two injections and $11(12 \%)$ eyes required three injections. During the second year, $23(26 \%)$ eyes required no additional injection, 30 (34\%) eyes required one injection, $26(29 \%)$ eyes required two injections, $8(9 \%)$ eyes required three injections and $2(2 \%)$ eyes required four injections. The total numbers of injection throughout the 2-year follow-up period were 1 (first injection only) in $7(8 \%)$ eyes, 2 in $12(13 \%)$ eyes, 3 in $13(15 \%)$ eyes, 4 in $28(31 \%)$ eyes, 5 in 17 (19\%) eyes, 6 in $10(11 \%)$ eyes and 7 in $2(2 \%)$ eyes. The number of reinjections tended to vary among the eyes. Rescue laser therapy was applied in $23(26 \%)$ eyes.

Figure 1 shows the changes in the mean logMAR VA during the 2-year follow-up period after the first bevacizumab injection. The baseline $\log$ MAR VA was $0.64 \pm 0.24$, which significantly improved 1 month after the first injection to $0.39 \pm 0.22$, and the VA improvement remained $0.33 \pm 0.211$ year after the first injection. These values were significantly $(p=0.001$ for both comparisons by the paired t test) better than the baseline VA. Although the VA 3 months after the first injection $(0.45 \pm 0.23)$ decreased from the VA level 1 month after the first injection because $59(66 \%)$ eyes had recurrent $\mathrm{ME}$ and required additional injections, the VA was still significantly $(p=0.001$ by the paired $t$ test) better than at baseline. During the second year, the improved VA remained $0.34 \pm 0.21$ at 2 years after the first injection ( $p=0.001$ by the paired $t$ test). Of the 89 study eyes, the $\log$ MAR VA 1 year after the first injection improved and decreased $0.3 \log$ MAR unit or more from baseline in $63(71 \%)$ eyes and 2 (2\%) eyes, respectively; the other $24(27 \%)$ eyes had less than a 0.3-unit change in the logMAR VA. Although the BRVO recurred during the second year in 2 of the 63 eyes with a VA improvement 1 year after the first injection, and the VA improvement was lost, the improved VA remained in the other 61 eyes 2 years after the first injection. Another two eyes with less than a 0.3 -unit change in the logMAR VA 1 year after the first injection had recurrent BRVO during the second year, resulting in VA loss. Thus, the logMAR VA 2 years after the first
Figure 1 The changes in the mean logarithm of minimum angle of resolution visual acuity (logMAR VA) during 2 years after the first injection of bevacizumab to treat macular oedema secondary to branch retinal vein occlusion. The vertical lines indicate $1 \mathrm{SE}$ of the mean. $\log \mathrm{MAR}$ VAs 1 month after the first injection and thereafter throughout the 2-year follow-up period significantly $(p=0.001$ for each comparison by the paired $t$ test) improved compared with the baseline logMAR VA.

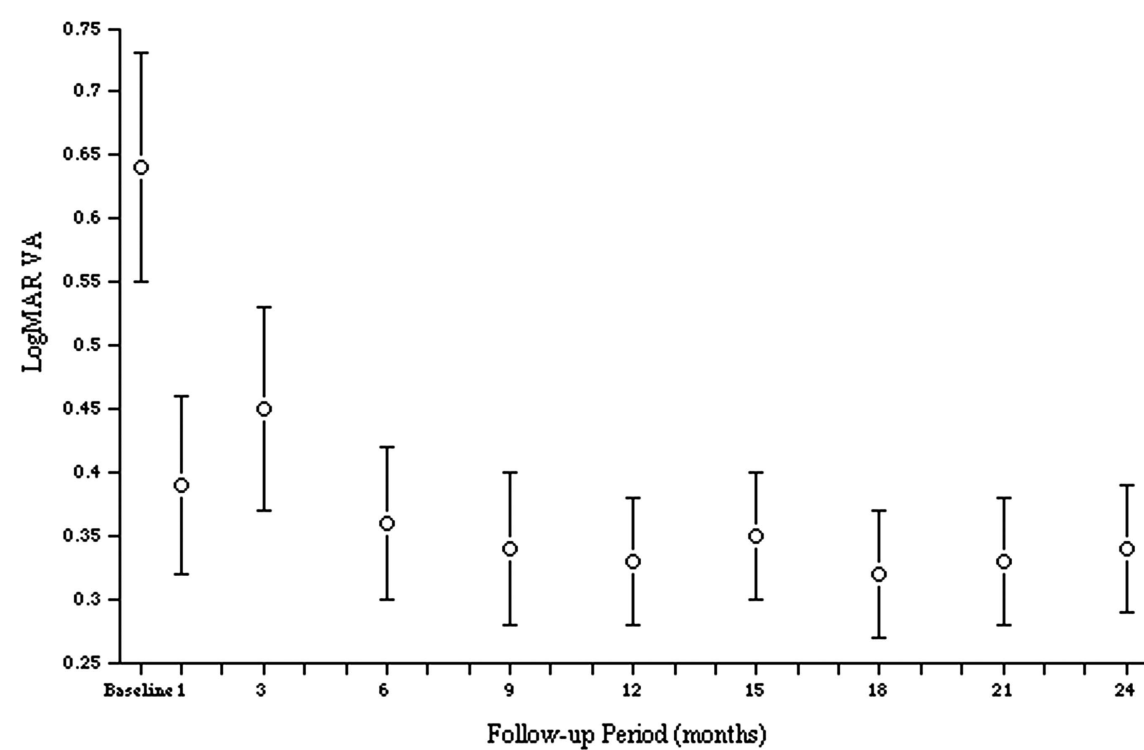


injection improved or decreased $0.3 \log$ MAR unit or more from baseline in $61(69 \%)$ eyes and $6(7 \%)$ eyes, respectively. The other $22(26 \%)$ eyes had less than a 0.3 -unit change in the $\log$ MAR VA. A VA of $20 / 40$ or better is considered a clinically relevant outcome because it is sufficient for reading and is the minimal VA required for driving. ${ }^{19}$ The percentage of eyes with a decimal VA of 0.5 (20/40 Snellen VA) or better was 4\% (4 eyes) at baseline, which increased to $66 \%$ (59 eyes) 2 years after the first injection. Of the eyes with a decimal VA of 0.5 or better, no eyes at baseline and 18 eyes 2 years after the first injection had a decimal VA of 1.0 (20/20 Snellen VA) or better. A VA of $20 / 200$ or worse (legal blindness) is a poor visual outcome. $^{20}$ This outcome occurred at 2 years after the first injection in $4(4 \%)$ eyes and was lower compared with baseline in $14(16 \%)$ eyes.

The mean foveal thickness 1 month after the first intravitreal injection $(212 \pm 77 \mu \mathrm{m})$ significantly improved compared with the mean foveal thickness at baseline $(572 \pm 134 \mu \mathrm{m})(\mathrm{p}=0.001$ by the paired $t$ test); thereafter, the mean foveal thickness improved and remained at $211 \pm 45 \mu \mathrm{m}$ at 1 year after the first injection ( $p=0.001$ by the paired $t$ test) (figure 2 ). Although the mean foveal thickness 3 months after the first injection (368 $\pm 126 \mu \mathrm{m})$ tended to increase, the foveal thickness still significantly ( $p=0.001$ by the paired $t$ test) improved compared with baseline. During the second year, the foveal thickness remained significantly $(\mathrm{p}=0.001$ by the paired $\mathrm{t}$ test $)$ improved at 220 $\pm 82 \mu \mathrm{m}$ at 2 years after the first injection.

In total, $58(67 \%)$ patients had systemic hypertension and 16 $(18 \%)$ patients had diabetes at baseline. The presence or absence of the diseases at baseline was not correlated with the baseline and final VAs.

No apparent ocular adverse events (endophthalmitis, uveitis, lens damage, cataract progression, prolonged IOP elevation) developed that were attributable to the bevacizumab injections. No eyes required cataract surgery during the 2-year follow-up period.

\section{DISCUSSION}

In the current study, the first IVB injection provided rapid visual and anatomic improvements in patients with ME secondary to
BRVO. The mean improvement in the logMAR VA was 0.25 at 1 month and a further improvement (0.31) occurred 1 year after the first injection, which was maintained throughout the second year (0.30 2 years after the first injection). In the Branch Retinal Vein Occlusion (BRAVO) study, ${ }^{78} 6$ monthly intraocular injections of $0.3 \mathrm{mg}$ or $0.5 \mathrm{mg}$ of ranibizumab provided rapid visual and anatomic improvements in patients with ME following BRVO. Beginning at month 6 after treatment and throughout the observation period, patients were examined monthly and could receive as-needed treatment, which resulted in a mean of 2.7 ( $0.5 \mathrm{mg}$ group) additional injections during the 6-month observation period. At month 12, the mean gain in the ETDRS letter score of 18.3 in the $0.5 \mathrm{mg}$ group after the initial 6 monthly ranibizumab injections was maintained. The HORIZON trial ${ }^{9}$ included patients with ME after BRVO who had completed the BRAVO study $^{8}$ and reported additional follow-up for a second year during which patients were evaluated every 3 months. This less stringent requirement for study visits resulted in fewer injections; however, the reduction in the frequency of ranibizumab injections during the second year of follow-up had little effect on the degree of the VA improvement. The degrees of the VA improvement in the current study were similar to those in the BRAVO $^{8}$ and HORIZON studies. ${ }^{9}$ Ranibizumab or bevacizumab for ME secondary to BRVO may have similar efficacy for improving the VA.

Although significant improvements in the ME secondary to BRVO have been reported after IVB injections, ${ }^{14-18}$ the optimal treatment protocol remains unknown. However, bevacizumab may not require monthly injections to achieve an optimal therapeutic response. ${ }^{17} 21$ An early report of the pharmacokinetics of intravitreal anti-VEGF agents in animal models suggested that bevacizumab has a longer intravitreal half-life than ranibizumab. In rabbits, the vitreous half-life of ranibizumab is 2.88 days versus 4.32 days for bevacizumab. ${ }^{22}$ Although there is no clinical evidence that patients receiving bevacizumab for retinal disease require less frequent injections than patients receiving ranibizumab, Epstein and coworkers ${ }^{23}$ achieved the same visual improvement in response to IVB injections administered every 6 weeks for central retinal vein occlusion as that obtained after
Figure 2 The changes in the mean foveal thickness during 2 years after the first injection of bevacizumab to treat macular oedema secondary to branch retinal vein occlusion. The vertical lines indicate $1 \mathrm{SE}$ of the mean. The foveal thicknesses 1 month after the first injection and thereafter throughout the 2-year follow-up period significantly $(p=0.001$ for each comparison by the paired t test) improved compared with the baseline foveal thickness.

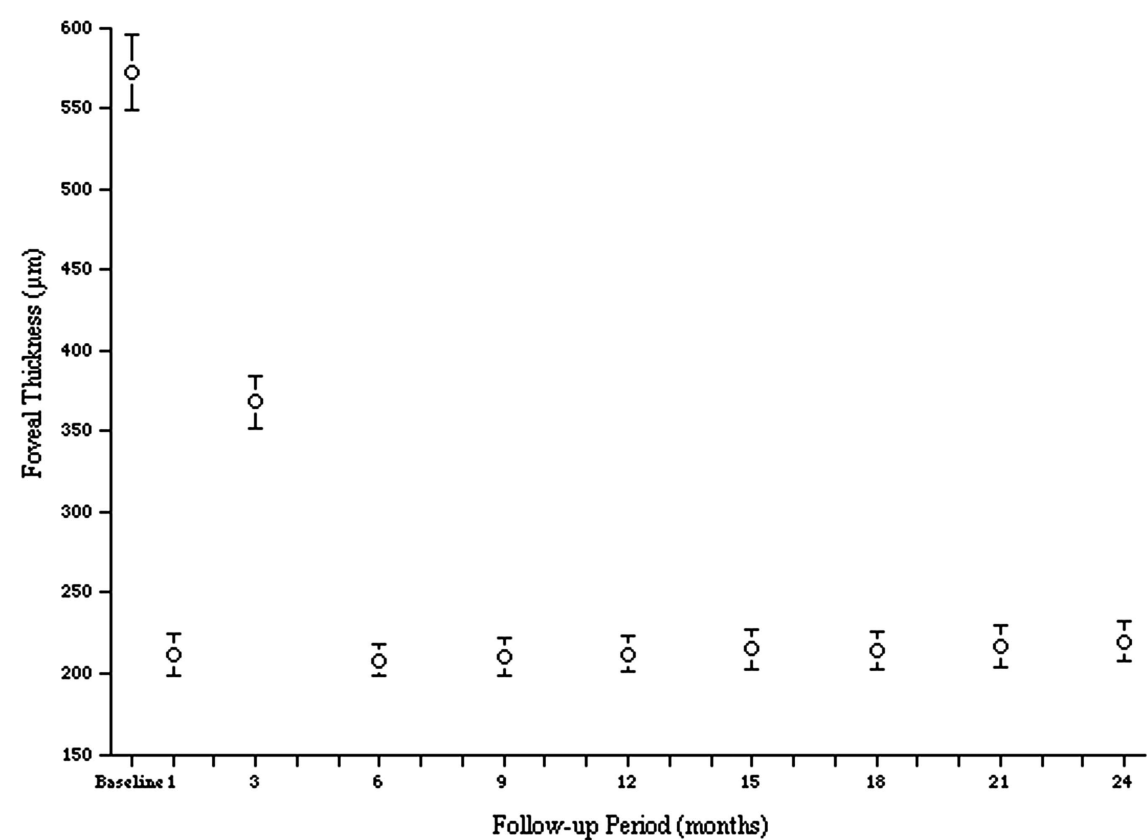


ranibizumab administered every 4 weeks in the Treatment of Macular Edema after Central Retinal Vein Occlusion study. ${ }^{24}$

The SCORE study group concluded that grid laser photocoagulation was the recommended therapy for ME secondary to BRVO. ${ }^{2}$ Thus, in the current study, rescue grid laser photocoagulation was offered to patients who met the protocolspecified treatment criteria. In the BRAVO trial, ${ }^{7}{ }^{8}$ rescue grid laser photocoagulation was offered once during the 6-month treatment period and once during the 6-month observation period for patients meeting the treatment criteria. At month 12 in the BRAVO study, ${ }^{8} 56.0 \%$ of patients in the $0.3 \mathrm{mg}$ group and $60.3 \%$ of patients in the $0.5 \mathrm{mg}$ group gained 15 ETDRS letters or more. In contrast, $20 \%$ of patients in the grid laser arm of the SCORE study ${ }^{2}$ gained 15 letters of VA at month 12 without concomitant anti-VEGF therapy. Although it is possible that part of the month 12 improvements in patients with BRVO was secondary to a concomitant laser effect, the immediate VA improvement was advantageous over laser therapy alone in patients with anti-VEGF therapy. Donati et al ${ }^{18}$ suggested that the efficacy of grid laser could reduce the number of intravitreal injections and maintain the long-term visual results. Since in the current study laser treatment was performed in eyes with a VA of 20/40 worse or those with fluorescein leakage even after IVB injections, no apparent differences in the VA outcomes and the number of IVB injections were found between eyes with and without laser treatment. However, we speculated that laser treatment may contribute to the reduction in the number of IVB injections in eyes with fluorescein leakage even after IVB injections.

In the current study, the mean total number of injections over 2 years was 3.8, including the first injection. This was lower compared with previous studies with different treatment protocols, for example, monthly injection of ranibizumab for 3 months ${ }^{12}$ or 6 months ${ }^{8}$ followed by as-needed reinjections. The longer intravitreal half-life of bevacizumab compared with ranibizumab may result in reduced reinjections. Donati et $a l^{18}$ reported that intravitreal bevacizumab with macular grid laser photocoagulation may reduce the number of reinjections. In the current study, we evaluated the macular condition using timedomain OCT, which has lower sensitivity for evaluating ME than spectral-domain OCT. Because of this lower sensitivity of time-domain OCT, which may miss macular cystic change and macular thickening imaged by spectral-domain OCT, physicians in this study may have treated some eyes less frequently, which may have otherwise recurrent ME on spectral-domain OCT. However, because of the excellent visual results in this study at 1 and 2 years, the current study supports a protocol of as-needed injections of bevacizumab combined with macular grid laser photocoagulation in the treatment of ME secondary to BRVO.

In the BRAVO study, ${ }^{7}$ there was continuous visual improvement with monthly ranibizumab injections up to month 3 with a significant improvement to month 6 and a continuous decrease in ME until month 3 with monthly injections. Immediate recovery of the physiologic macular morphology potentially minimises deterioration of the retinal function resulting from persistent or recurrent $\mathrm{ME}$ and consequently improves the visual function. Since in the current study ME recurred 3 months after the first IVB injection with an as-needed reinjection schedule, 3 monthly injections at the induction phase may further improve the VA outcomes. In the current study, the logMAR VA 2 years after the first injection decreased 0.3 logMAR unit or more from baseline in six (7\%) eyes and the VA was $20 / 200$ or worse 2 years after the first injection in four
(4\%) eyes. Although recurrent occlusion of the retinal branch vein during the second year resulted in a decrease of 0.3 unit or more in the logMAR VA in four eyes, recurrent or persistent $\mathrm{ME}$ resulting from an as-needed reinjection schedule may be associated with the above unfavourable VA outcomes.

The current study was not designed to evaluate the effect of disease duration on the likelihood of spontaneous resolution of ME secondary to BRVO. The possibility of spontaneous improvement should not rule out early treatment in eyes with ME secondary to BRVO. In fact, seven (8\%) eyes in the current study did not require additional IVB injections after the first injection. In those eyes, ME might have resolved during the natural disease course. However, no definitive data can predict the eyes in which ME will resolve during the natural disease course. The SCORE study ${ }^{2}$ found that a shorter duration of ME before treatment was associated with greater improvement in the retinal thickness with treatment.

The limitations of the current study included a lack of control groups and use of a Landolt ring VA chart. The results cannot be used to compare the effectiveness of bevacizumab and ranibizumab and follow-up regimens. Another limitation was a $15 \%$ (16/105 eyes) dropout rate during the 2-year follow-up period. More than 3 months had passed during the second year without recurrence of $\mathrm{ME}$ before the patients dropped out, and $\mathrm{ME}$ resolved after bevacizumab injection in four eyes. Since the reason for losing the patients during follow-up may be unrelated to a poor treatment response but to visual stabilisation, the possibility of underestimating the number of injections in the current study seems small. The variable follow-up of at least 3 months and the lack of a prospective determination of follow-up make this study more likely to be a retrospective review of treatment results with bevacizumab rather than a true prospective trial with prespecified study visits, which was another limitation of this study. The current large case series with a 2-year follow-up period showed the favourable outcomes of IVB injections for treating ME secondary to BRVO. Bevacizumab provides substantial long-term benefits to patients with ME secondary to BRVO, but multiple injections are required in some patients.

Contributors TH is responsible for the conception, design, acquisition of data, analysis, drafting and revising the article, and final approval of all versions of the manuscript. MH, TM, SK, RM, KT and $\mathrm{HO}$ are responsible for the conception, design and final approval of all versions of the manuscript. HK and SS are responsible for the interpretation of data and final approval of all versions of the manuscript.

Competing interests Dr Hikichi received lecture fees from Novartis Pharma Japan, Bayer Japan, Santen, and Alcon Japan. No other authors have any conflicts of interest to declare.

Ethics approval The study protocol was approved by the institutional review board of Ohtsuka Eye Hospital before study initiation.

Provenance and peer review Not commissioned; externally peer reviewed.

Open Access This is an Open Access article distributed in accordance with the Creative Commons Attribution Non Commercial (CC BY-NC 3.0) license, which permits others to distribute, remix, adapt, build upon this work non-commercially, and license their derivative works on different terms, provided the original work is properly cited and the use is non-commercial. See: http://creativecommons.org/ licenses/by-nc/3.0/

\section{REFERENCES}

1 Branch Vein Occlusion Study Group. Argon laser photocoagulation for macular edema in branch vein occlusion. Am J Ophthalmol 1984:98:271-82.

2 SCORE Study Research Group. A randomized trial comparing the efficacy and safety of intravitreal triamcinolone with standard care to treat vision loss associated with macular edema secondary to branch retinal vein occlusion: the Standard Care vs. Corticosteroid for Retinal Vein Occlusion (SCORE) Study report 6. Arch Ophthalmol 2009;127:1115-28 
3 Early Treatment Diabetic Retinopathy Study Research Group. Photocoagulation for diabetic macular edema: early Treatment Diabetic Retinopathy Study report number 1. Arch Ophthalmol 1985;103:1796-806.

4 Campochiaro PA, Hafiz G, Shah SM, et al. Ranibizumab for macular edema due to retinal vein occlusions: implication of VEGF as a critical stimulator. Mol Ther 2008;16:791-9.

5 Noma H, Funatsu H, Yamasaki M, et al. Pathogenesis of macular edema with branch retinal vein occlusion and intraocular levels of vascular endothelial growth factor and interleukin-6. Am J Ophthalmol 2005;140:256-61.

6 Park SP, Ahn JK, Mun GH. Aqueous vascular endothelial growth factor levels are associated with serous macular detachment secondary to branch retinal vein occlusion. Retina 2010;30:281-6.

7 Campochiaro PA, Heier JS, Feiner L, et al. Ranibizumab for macular edema following branch retinal vein occlusion: six-month primary end point results of a phase III study. Ophthalmology 2010;117:1102-12.

8 Brown DM, Campochiaro PA, Bhisitkul RB, et al. Sustained benefits from ranibizumab for macular edema following branch retinal vein occlusion: 12-month outcomes of a phase III study. Ophthalmology 2011;118:1594-602.

9 Heier JS, Campochiaro PA, Yau L, et al. Ranibizumab for macular edema due to retinal vein occlusions, long-term follow-up in the HORIZON trial. Ophthalmology 2012;119:802-9.

10 Hahn P, Fekrat S. Best practices for treatment of retinal vein occlusion. Curr Opin Ophthalmol 2012;23:175-81.

11 Pece A, Isola V, Piermarocchi S, et al. Efficacy and safety of anti-vascular endothelial growth factor (VEGF) therapy with intravitreal ranibizumab (Lucentis) for naive retinal vein occlusion: 1-year follow-up. Br J Ophthalmol 2011;95:56-68.

12 Campochiaro PA, Hafiz G, Channa R, et al. Antagonism of vascular endothelial growth factor for macular edema caused by retinal vein occlusions: Two-year outcomes. Ophthalmology 2010;117:2387-94.

13 Figueroa MS, Contreras I, Noval S, et al. Results of bevacizumab as the primary treatment for retinal vein occlusions. Br J Ophthalmol 2010;94:1052-6.
14 Prager F, Michels S, Kriechbaum K, et al. Intravitreal bevacizumab (Avastin) for macular oedema secondary to retinal vein occlusion: 12-month results of a prospective clinical trial. Br J Ophthalmol 2009:93:452-6.

15 Kreutzer TC, Alge CS, Wolf AH, et al. Intravitreal bevacizumab for the treatment of macular oedema secondary to branch retinal vein occlusion. $\mathrm{Br} J$ Ophthalmol 2008;92:351-5.

16 Prager F, Michels S, Kreiechbaum K, et al. Intravitreal bevacizumab (Avastin) for macular oedema secondary to retinal vein occlusion: 12-month results of a prospective clinical trial. Br J Ophthalmol 2009;93:452-6.

17 Ehlers J, Decroos FC, Fekrat S. Intravitreal bevacizumab for macular edema secondary to branch retinal vein occlusion. Retina 2011;31:1856-62.

18 Donati S, Barosl P, Bianchi M, et al. Combined intravitreal bevacizumab and grid laser photocoagulation for macular edema secondary to branch retinal vein occlusion. Eur J Ophthalmol 2012;22:607-14.

19 American Medical Association. Physician's guide to assessing and counseling older drivers. 2nd edn. Chicago: American Medical Association, 2010:69-140. http:// www.ama-assn.org/resources/doc/publichealth/older-drivers-guide.pdf

20 National Eye Institute. National plan for eye and vision research. Low vision and blindness rehabilitation. Bethesda: US Department of Health and Human Services, 2011. http://www.nei.nih.gov/strategicplanning/np_low.asp

21 Hoeh AE, Ach T, Schaal KB, et al. Long-term follow-up of OCT-guided bevacizumab treatment of macular edema due to retinal vein occlusion. Graefes Arch Clin Exp Ophthalmol 2009;247:1635-41.

22 Bakri SJ, Snyder MR, Reid JM, et al. Pharmacokinetics of intravitreal ranibizumab (Lucentis). Ophthalmology 2007;114:2179-82.

23 Epstein DL, Algvere PV, von Wendt G, et al. Bevacizumab for macular edema in central retinal vein occlusion: a prospective, randomized, double-masked clinical study. Ophthalmology 2012;119:1184-9.

24 Brown DM, Campochiaro PA, Signh RP, et al. the CRUISE investigators. Ranibizumab for macular edema following central retinal vein occlusion: six-month primary end point results of a phase III study. Ophthalmology 2010;117:1124-33. 